\title{
Comparison of the cost, choice and availability of a healthy balanced gluten-free diet (GFD) with a standard diet that meet nutrient and food based guidance
}

\author{
G. Abernethy and E. Bannerman \\ Queen Margaret University, Queen Margaret University Drive, Musselburgh, East Lothian, EH21 6UU, UK
}

Coeliac disease (CD) is a chronic autoimmune inflammatory disorder of the small intestine; an immunological response to ingested gluten. Management requires diagnosed individuals to adhere to a strict gluten-free diet (GFD). Adherence to a GFD is known to be affected by factors such as cost ${ }^{(1)}$, with some GF items seven times more expensive than standard gluten-containing products ${ }^{(2)}$. Access to healthier food choices has been shown to be limited in more rural localities and also in areas of greater deprivation, although size of retail outlet is likely to be a key factor ${ }^{(3)}$. This study aims to assess the cost, choice and availability of a nutritionally balanced GF $v$. standard market basket in supermarkets in four different localities in Scotland accounting for high and low SES and also rural and urban locations.

A market basket of foods for meals for a week was developed to meet dietary reference values, nutrient recommendations ${ }^{(4)}$ and food based guidelines for adults in the $\mathrm{UK}^{(5)}$, and was based on foods consumed within the NDNS ${ }^{(6)}$. The cost, choice and availability of both the standard market basket and a GF-basket items was determined in four supermarkets in differing locations in Scotland. The Scottish Index of Multiple Deprivation ${ }^{(7)}$ was used to identify four locations that represent the extremes of socio-economic status and also locality (urban and rural) settings. Data collection noted cost of the cheapest and most expensive food items per portion for 'branded' and 'ownbrand' products. A 12 months pre-paid prescription charge of $£ 28$ was assumed to account for the use of prescribed GF products. The number of GF food items $v$. gluten containing items was reported as per 'The Eat-well Plate' food groups ${ }^{(8)}$.

The cost of the average GF basket (mean £49.71) was significantly more expensive than the average standard basket (mean diff $=£ 7.50 ; \mathrm{U}=71.0 \mathrm{P}=0.032$ ). A GF basket made up using 'own brand' products was cheaper than GF basket using 'branded' products. There was less choice and availability of gluten-free products (GFP) compared with standard foods, particularly in rural areas. Across all supermarkets foods high in fats/sugars were more readily available compared with other food groups.

This study established that a nutritionally-balanced GF market basket, based on foods typically eaten by the UK population, costs more than a standard market basket and also more than the average weekly spend on food ${ }^{(9)}$. Inequalities are likely to exist in accessing a healthy GFD diet in different localities across Scotland. Whether this affects nutritional quality and dietary adherence needs further study.

1. Lee AR, Ng DL, Zivin J et al. (2007) J Hum Nutr Diet 20, 423-430.

2. Coeliac UK (2010) 'Coeliac UK Cost Project 2010' Crossed Grain, 77, 22-23.

3. Dawson J, Marshall D, Taylor M et al. (2008) Accessing Healthy Food. FSA Scotland.

4. DH (1991) Dietary Reference Values for Food Energy and Nutrients in the United Kingdom. London: HMSO.

5. NHS Choices (2010) Livewell: Eight Tips for Healthy eating. London: NHS.

6. Hoare J, Henderson L, Bates CJ et al. (2004) The NDNS: Adults Aged 19-64 Year. Vol. 5. London: TSO.

7. Scottish Government (2009) Scottish Index of Multiple Deprivations. Edinburgh: Information Services Division.

8. FSA (2007) The Eat-well Plate. London: FSA.

9. DEFRA (2007) UK Purchases \& Expenditure on Food \& Drink \& derived Energy \& Nutrient Intakes in 2007. 